\title{
Preoperative inflammation-based scores predict early recurrence after lung cancer resection
}

\author{
Katsuya Watanabe $^{1 \wedge}$, Daisuke Noma ${ }^{1,2}$, Haruhiko Masuda $^{1}$, Munetaka Masuda $^{3}$ \\ ${ }^{1}$ Department of General Thoracic Surgery, National Hospital Organization Yokohama Medical Center, Yokohama, Japan; ${ }^{2}$ Department of General \\ Thoracic Surgery, Yokohamashi Nanbu Hospital, Yokohama, Japan; ${ }^{3}$ Department of Surgery, Yokohama City University, Yokohama, Japan \\ Contributions: (I) Conception and design: K Watanabe; (II) Administrative support: M Masuda; (III) Provision of study materials or patients: K \\ Watanabe, D Noma, H Masuda; (IV) Collection and assembly of data: D Noma, H Masuda; (V) Data analysis and interpretation: K Watanabe, D \\ Noma; (VI) Manuscript writing: All authors; (VII) Final approval of manuscript: All authors. \\ Correspondence to: Katsuya Watanabe, PhD. Department of General Thoracic Surgery, National Hospital Organization Yokohama Medical Center, \\ Yokohama, Japan, 3-60-2, Harajuku, Totsuka Ward, Yokohama, Kanagawa 245-8575, Japan. Email: watanabe.katsuya.gt@mail.hosp.go.jp.
}

\begin{abstract}
Background: Inflammation in the tumor microenvironment is hypothesized to have a major role in cancer invasiveness, progression, and metastases. The purpose of this study was to evaluate the prognostic value of preoperative inflammation-based scores in terms of estimating the timing of recurrence by hazard curves in a cohort of operable, early-stage non-small cell lung cancer (NSCLC) patients.

Methods: A total of 387 patients with NSCLC who underwent complete pulmonary resection from 2010 to 2019 had their C-reactive protein-to-albumin ratio (CAR), neutrophil-to-lymphocyte ratio (NLR), plateletto-lymphocyte ratio (PLR), Glasgow prognostic score (GPS), modified GPS, systemic immune-inflammation index (SII), and advance lung cancer inflammation index (ALI) measurements taken before surgery. Hazard curves indicating changes in hazards over time were evaluated.

Results: Median follow-up was 39.2 months. In total, 105 patients (27.1\%) experienced recurrence. The resulting hazard curve with elevated CAR, SII, GPS, and mGPS, values displayed an initial high peak during the first year. Multivariate analyses showed that an elevated CAR [hazard ratio (HR), 1.987; 95\% confidence interval (CI), 1.202-3.284] independently predicted the recurrence-free survival. Even in stage I disease, patients with elevated CAR and SII values showed an earlier peak of recurrence, which was about 12 to 16 months earlier than those with low values.

Conclusions: Even after complete resection of stage I NSCLC, patients with elevated CAR and SII values retain a high risk of early recurrence. Preoperative inflammation-based scores can be an objective, simple, and cost-effective measurement for predicting early recurrence of NSCLC.
\end{abstract}

Keywords: Non-small cell lung cancer (NSCLC); inflammation-based scores; hazard curve; recurrence; postoperative follow-up

Submitted Dec 06, 2020. Accepted for publication Mar 18, 2021.

doi: $10.21037 /$ jtd-20-3458

View this article at: http://dx.doi.org/10.21037/jtd-20-3458

\section{Introduction}

Lung cancer has been reported to be the leading cause of cancer mortality worldwide, and approximately $85 \%$ of primary lung cancer diagnoses are non-small cell lung cancer (NSCLC) (1). Even though surgery remains the mainstay of treatment for NSCLC, early recurrence often occurs even in patients who undergo curative-intent resection, early recurrence often develops even in patients

\footnotetext{
^ ORCID: 0000-0001-8898-0370.
} 
with early disease who undergo complete resection, and the recurrence risk in NSCLC varies throughout the follow-up duration $(2,3)$.

Recently, it has been widely recognized that systemic inflammatory responses substantially contribute to tumor development, tumor invasion, metastatic spread, and enhancement of local immunosuppression (4-7), and it has been reported that systemic inflammation and malnutrition are correlated with a poor prognosis in various cancers. In addition, inflammation-based scores, including the C-reactive protein/albumin ratio (CAR) $(8,9)$, neutrophilto-lymphocyte ratio (NLR) (10,11), platelet-to-lymphocyte ratio (PLR) (12,13), Glasgow prognostic score (GPS) $(14,15)$, modified GPS (mGPS) $(16,17)$, Systemic immuneinflammation index (SII) $(18,19)$, and advanced lung cancer inflammation index (ALI) (20) have been validated in NSCLC.

Although these previous studies simply analyzed the total risks of events using cumulative incidence curves plotted by the Kaplan-Meier method, such curves do not provide direct information on changes in event-specific hazard rates during the follow-up period. To our knowledge, the relationship between the timing of recurrence and inflammation-based scores has never been evaluated using hazard curves. It is necessary not only to determine the total risk of recurrence but also to ascertain when recurrence is most likely to develop. In addition, to understand how the preoperative inflammation-based scores are related to changes in the recurrence risk over time would be beneficial to achieve a more efficient follow-up surveillance.

Therefore, in the present study, we evaluated the influence of inflammation-based scores on the timing of recurrence after complete resection for NSCLC.

We present the following article in accordance with the REMARK reporting checklist (available at http://dx.doi. org/10.21037/jtd-20-3458).

\section{Methods}

\section{Study design}

This study was conducted in accordance with the Declaration of Helsinki (as revised in 2013). The study was approved by the institutional ethics board of National Hospital Organization Yokohama Medical Center (No. 2019-11), and individual consent for this retrospective analysis was waived. The patients' records of 448 subsets who underwent complete resection of NSCLC from
January 2010 to December 2019 were investigated. Patients who died during the immediate postoperative period, those with recurrence within 3 months after surgery, those with acute infection (e.g., empyema, pneumonia), those with active double cancer, those who underwent induction chemotherapy, those with history of surgery for NSCLC, and those taking corticosteroids or immunosuppressive drugs were excluded. Finally, 387 patients (233 men, 154 women) with a single primary tumor were enrolled in this study (Figure 1).

Routine preoperative staging investigations were performed using high-resolution computed tomography (HRCT) of the chest and abdomen combined with brain imaging, bone scintigraphy or positron emission tomography (PET), and tumors were staged according to the tumor, lymph node, and metastatic Classification of Malignant Tumors, seventh edition (21). A single primary tumor was diagnosed in all patients, and no patients had a history of lung cancer (excluding those with multicentric cancers).

\section{Definition of inflammation-based scores}

GPS was calculated based on the CRP and albumin values. Patients with high CRP levels $(>1.0 \mathrm{mg} / \mathrm{dL})$ and hypoalbuminemia $(<3.5 \mathrm{~g} / \mathrm{dL})$ were assigned a score of 2 , while those with high CRP levels $(>1.0 \mathrm{mg} / \mathrm{dL})$ or hypoalbuminemia $(<3.5 \mathrm{~g} / \mathrm{dL})$ were assigned a score of 1 , and those with normal CRP and albumin levels were assigned a score of 0 . Regarding modified GPS (mGPS), patients with high CRP $(>0.3 \mathrm{mg} / \mathrm{dL})$ and hypoalbuminemia $(<3.5 \mathrm{~g} / \mathrm{dL})$ were assigned a score of 2 , while those with only a high CRP level were assigned a score of 1 , and those with a normal CRP level were a assigned a score of 0 , irrespective of the albumin values. The CAR was calculated from the serum CRP value divided by the serum albumin value. The SII was calculated as neutrophils $\times$ platelets/lymphocytes, and the NLR was simply determined as the number of neutrophils divided by the number of lymphocytes. The PLR was defined as the number of platelets divided by the number of lymphocytes, and the ALI was calculated as the product of the body mass index (BMI) and albumin divided by the NLR. All inflammation-based scores were calculated from the preoperative routine laboratory data.

\section{Patient follow-up}

Follow-up evaluations included physical examination, 


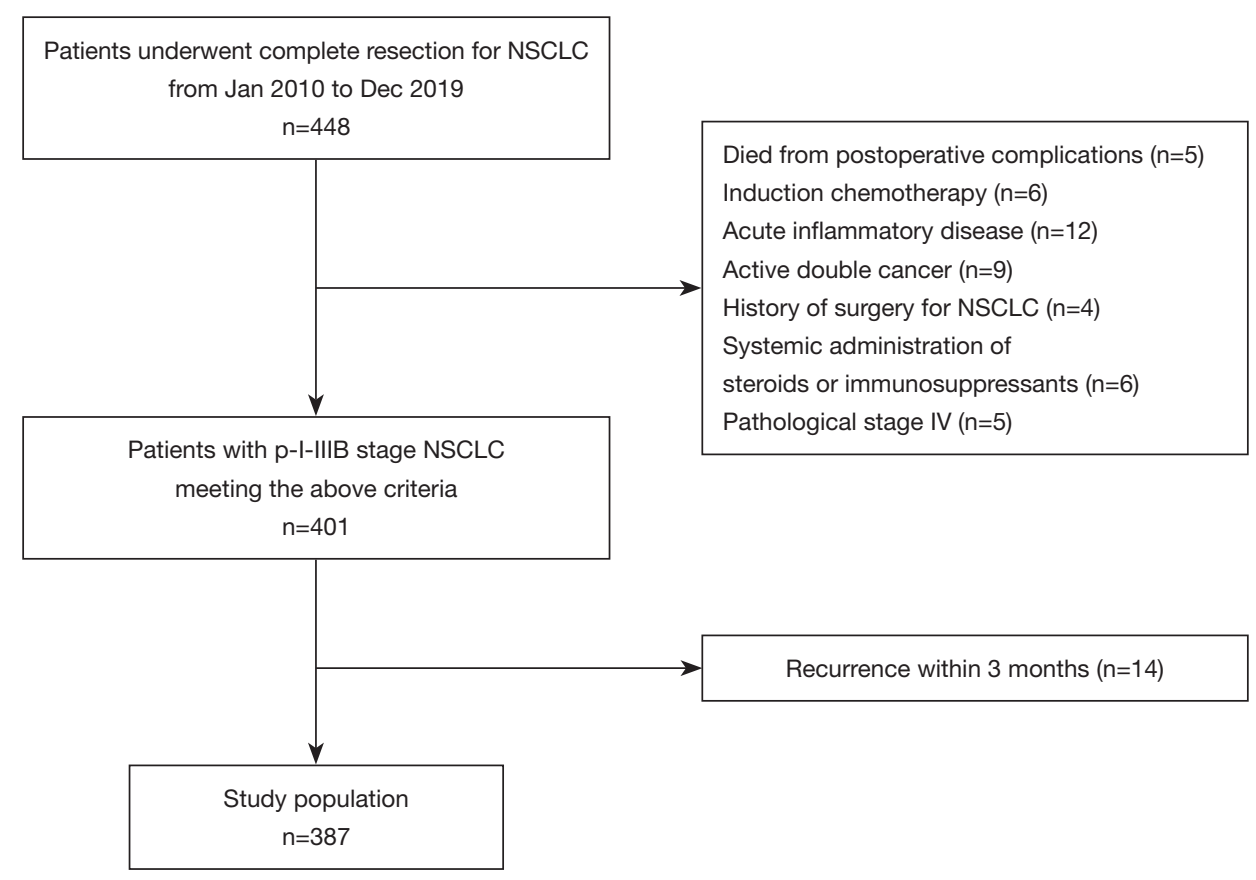

Figure 1 Flowchart of the study population inclusion. NSCLC, non-small cell lung cancer.

chest radiography, and CT scanning of the chest and abdomen. In general, CT was performed every six months in the first two years after surgery and annually thereafter. Additional imaging studies including CT, brain MRI, bone scintigraphy, and PET were performed for patients with any signs or symptoms of recurrence as required.

Recurrence was diagnosed with the assistance of a radiooncologist based on the findings of a physical examination and diagnostic imaging findings, and it was histologically or cytologically confirmed if necessary. Cases with second primary lung cancers defined as a single lung lesion occurring after a prior resection $(22,23)$ were excluded.

\section{Statistical analyses}

Continuous variables were presented as the mean and standard deviation, while categorical variables were presented as the frequencies and percentage. Two-category comparisons were performed by Student's $t$-test and Fisher's exact test.

The recurrence-free survival (RFS) was defined as the interval between the date of surgery to the date of recurrence or death, and patients without any events were censored on the date of the last follow-up. The RFS was analyzed using the Kaplan-Meier method with the log-rank test. The muhaz package for smoothing a hazard function for censored data proposed by Müller and Wang (24) was used to estimate the hazard curves. The cut-off values of the CAR, NLR, PLR, SII, and ALI to test the predictive ability for disease recurrence were determined by a receiver operating characteristic (ROC) curve analysis. A multivariate Cox regression analysis adjusting for the age, sex, smoking status, BMI, surgery type, surgical procedure, and pathological stage was performed to evaluate the relationship between inflammation-based scores and the RFS. All analyses were conducted using R 3.6.2 (R Foundation for Computing, Vienna, Austria) software programs. $\mathrm{P}$ values of less than 0.05 were considered to indicate statistical significance. A professional statistician reviewed and verified the statistical analyses.

\section{Results}

\section{Clinicopathological features}

The characteristics of the patients are summarized in Table 1 . The median follow-up period for the 387 patients was 39.2 (range, 3 to 117) months, and the median age was 71 (range, 19 to 86 ) years old.

The optimal thresholds for the CAR, NLR, PLR, SII, and ALI based on ROC curve analyses were set at 0.014 , $2.90,231,715$, and 37 , respectively. 
Table 1 Clinicopathological characteristics

\begin{tabular}{|c|c|}
\hline Variables & No. $(\%)$ or mean \pm SD (range) \\
\hline Age & $70.6 \pm 9.4(19-86)$ \\
\hline \multicolumn{2}{|l|}{ Gender } \\
\hline Male & $233(60.2)$ \\
\hline Female & $154(39.8)$ \\
\hline Body mass index, $\mathrm{kg} / \mathrm{m}^{2}$ & $22.5 \pm 3.4$ \\
\hline$<18.5^{\mathrm{a}}$ & $35(9.0)$ \\
\hline $18.5-25.0^{b}$ & $271(70.3)$ \\
\hline$\geq 25.0^{c}$ & $81(20.9)$ \\
\hline \multicolumn{2}{|l|}{ Smoking status } \\
\hline Never smoker & $128(33.1)$ \\
\hline Light smoker (<30 PY) & $81(20.9)$ \\
\hline Heavy smoker ( $\geq 30 \mathrm{PY})$ & $178(46.0)$ \\
\hline CAR & $0.12 \pm 0.4$ \\
\hline NLR & $2.41 \pm 1.3$ \\
\hline PLR & $152.1 \pm 71.9$ \\
\hline SII & $587.3 \pm 413.3$ \\
\hline ALI & $46.4 \pm 24.2$ \\
\hline \multicolumn{2}{|l|}{ GPS } \\
\hline 0 & $336(86.8)$ \\
\hline 1 & $34(8.8)$ \\
\hline 2 & $17(4.4)$ \\
\hline \multicolumn{2}{|l|}{ mGPS } \\
\hline 0 & 303 (78.3) \\
\hline 1 & $63(16.3)$ \\
\hline 2 & $21(5.4)$ \\
\hline \multicolumn{2}{|l|}{ Surgery type } \\
\hline VATS & $324(83.7)$ \\
\hline Open & $63(16.3)$ \\
\hline \multicolumn{2}{|l|}{ Surgical procedure } \\
\hline Wedge resection & $50(12.9)$ \\
\hline Segmentectomy & $31(8.0)$ \\
\hline Lobectomy & $292(75.5)$ \\
\hline Pneumonectomy & $14(3.6)$ \\
\hline
\end{tabular}

Table 1 (continued)
Table 1 (continued)

\begin{tabular}{lc}
\hline Variables & No. (\%) or mean \pm SD (range) \\
\hline Histological type & $283(73.1)$ \\
Adenocarcinoma & $85(22.0)$ \\
Squamous cell carcinoma & $7(1.8)$ \\
Adenosquamous carcinoma & $12(3.1)$ \\
Others & \\
Pathological stage & $189(48.8)$ \\
IA & $73(18.9)$ \\
IB & $39(10.1)$ \\
IIA & $32(8.3)$ \\
IIB & $50(12.9)$ \\
IIIA & $4(1.0)$ \\
IIIB & \\
Adjuvant chemotherapy & $280(72.4)$ \\
No & $107(27.7)$ \\
Yes &
\end{tabular}

${ }^{a}$, underweight; ${ }^{b}$, normal weight; ${ }^{c}$, overweight and obesity. Alb, albumin; ALI, advanced lung cancer inflammation index; BMI, body mass index; CAR, the C-reactive protein/albumin ratio; GPS, Glasgow prognostic score; mGPS, modified GPS; NLR, neutrophil-lymphocyte ratio; PLR, platelet lymphocyte ratio; PY, pack-year; SII, the Systemic immune-inflammation index; SD, standard deviation; VATS, video-assisted thoracoscopic surgery.

\section{Survival analysis findings}

One hundred and five (27.1\%) of the 387 patients experienced recurrence (local recurrence in 43 patients and either distant metastasis alone or both local recurrence and distant metastasis in 62 patients). Figures 2 and 3 show the Kaplan-Meier survival curves of recurrence after surgery for each inflammationbased score. Patients with elevated CAR, SII, and mGPS values had a significantly poorer RFS than those with lower values. The resulting hazard curves in patients with elevated CAR and SII values showed an initial sharp and high peak within one year after surgery, indicative of early recurrence. In contrast, the peak of patients with low values had a relatively wide gentle slope (Figure 4).

A comparison of inflammation-based scores in cases with pathological stage I and stage IIA or higher disease showed 
A

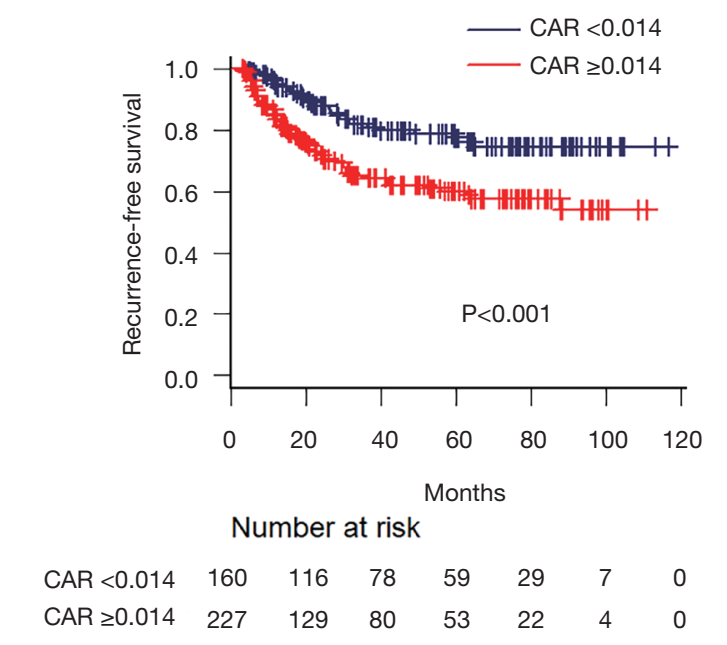

B

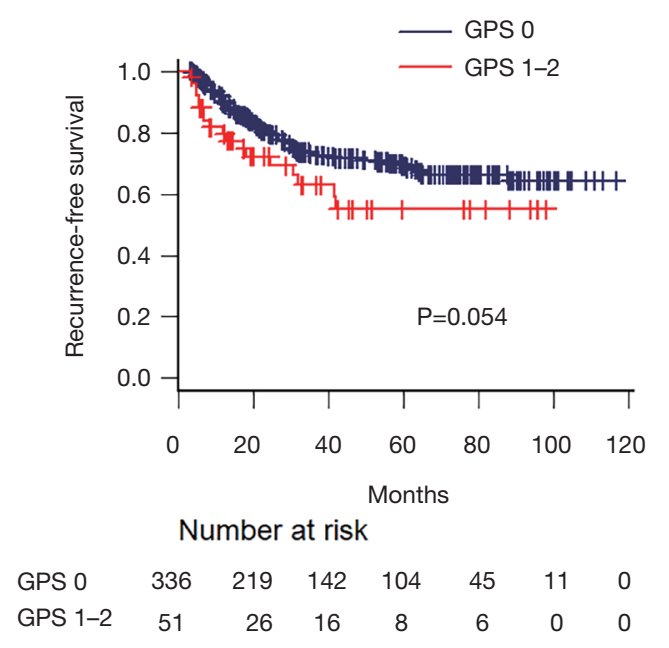

C
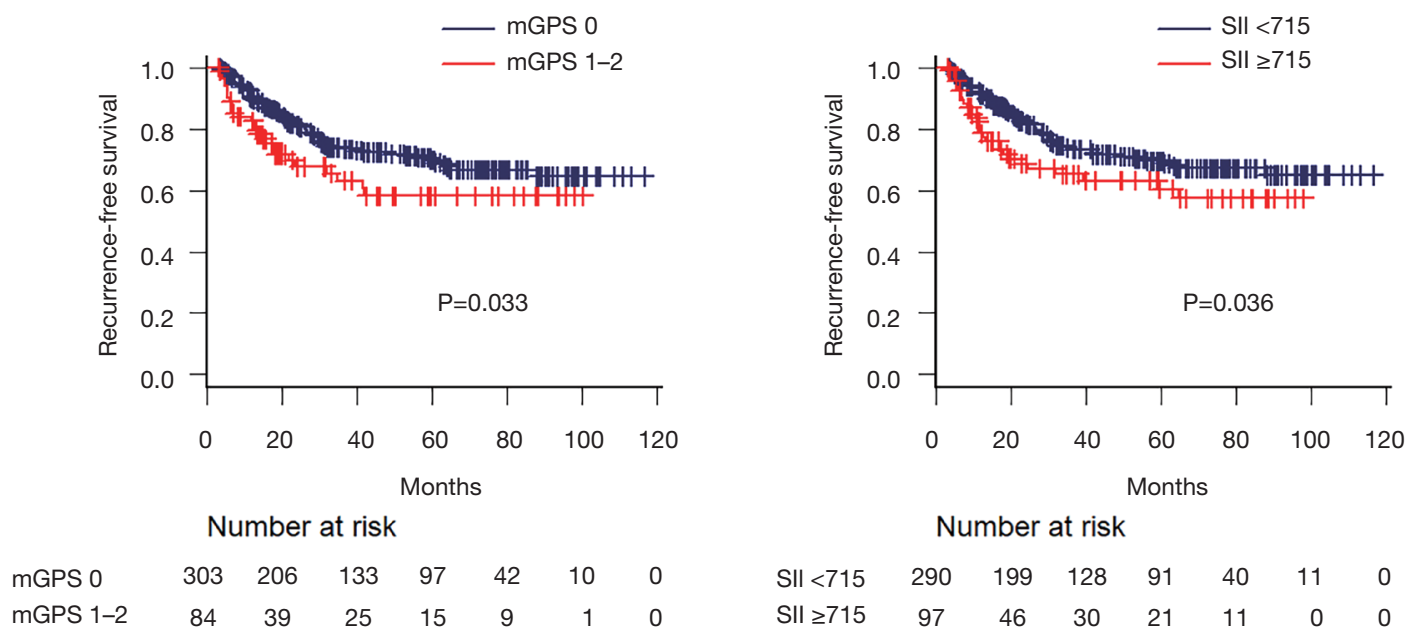

Figure 2 Kaplan-Meier curves for recurrence-free survival curves according to inflammation-based scores. (A) CAR; (B) GPS; (C) mGPS; (D) SII. CAR, C-reactive protein-to-albumin ratio; GPS, Glasgow prognostic score; mGPS, modified GPS; SII, Systemic immune-inflammation index.

that patients with elevated CAR, SII, and mGPS values were significantly more common in patients with advancedstage NSCLC than in those with stage I NSCLC (Table 2).

According to a multivariate analysis of the RFS, the CAR $(\mathrm{P}=0.007)$ was found to be an independent prognostic factor (Table 3).

The hazard curves of CAR and SII according to the pathological stage (Figure 5) were shown to be similar (highest peak around one year after surgery) for both patients with elevated values and those with low values in patients with stage IIA or higher (Figure $5 B, D$ ). In patients with stage I, the hazard rate curve showed an initial high peak around 12 months after surgery for patients with elevated CAR and SII values (Figure $5 A, C$ ). In contrast, patients with low values had a relatively wide gentle slope, which peaked about 12 to 16 months later than the peak in those with elevated CAR and SII values.

\section{Discussion}

In the present study, we assessed the prognostic value of inflammation-based scores in terms of estimating the 
A

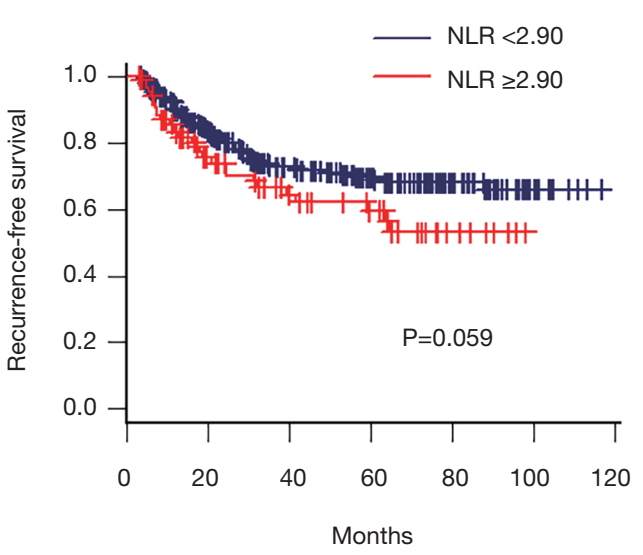

Number at risk
B

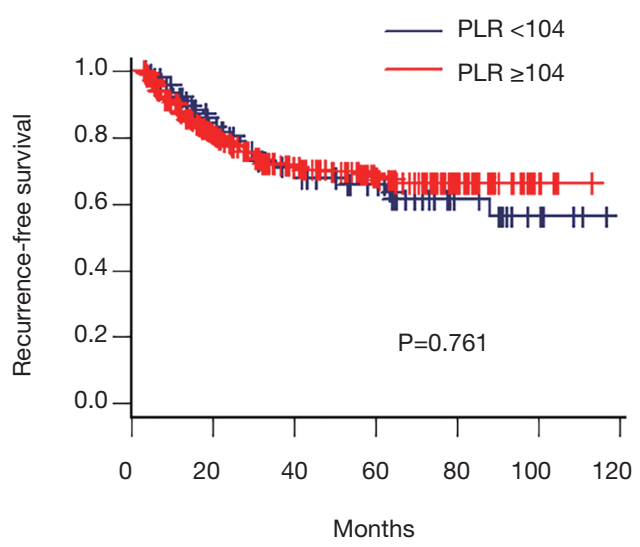

Number at risk

$\begin{array}{lccccccc}\text { NLR }<2.90 & 298 & 199 & 129 & 91 & 44 & 11 & 0 \\ \text { NLR } \geq 2.90 & 89 & 46 & 29 & 21 & 7 & 0 & 0\end{array}$

PLR $<104$

$\begin{array}{ccccccc}91 & 63 & 42 & 32 & 13 & 5 & 0 \\ 296 & 182 & 116 & 80 & 38 & 6 & 0\end{array}$

C

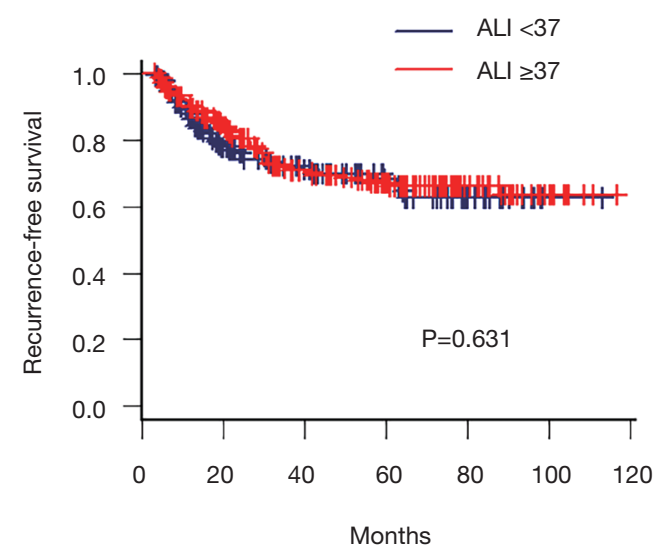
Number at risk

$\begin{array}{lccccccc}\text { ALI }<37 & 165 & 93 & 59 & 39 & 16 & 1 & 0 \\ \text { ALI } \geq 37 & 222 & 152 & 99 & 73 & 35 & 10 & 0\end{array}$

Figure 3 Kaplan-Meier curves for recurrence-free survival according to inflammation-based scores. (A) NLR; (B) PLR; (C) ALI. NLR, neutrophil-to-lymphocyte ratio; PLR, platelet-to-lymphocyte ratio; ALI, advance lung cancer inflammation index.

timing of recurrence by hazard curves in patients with completely resected NSCLC. Our results indicated that patients with elevated CAR, SII, and mGPS values showed a significantly worse RFS than those with low values. In addition, resulting hazard curves for patients with elevated CAR and SII values showed an earlier and higher maximum peak than in patients with low values. Furthermore, the CAR was an independent predictor of the RFS, and patients with elevated CAR and SII values retained a high risk of early recurrence, even in cases of completely resected stage I NSCLC.

To our knowledge, this is the first report to describe a relationship between the timing of recurrence and inflammation-based scores for resected NSCLC patients using hazard curve. Since the time of Virchow, inflammation has been linked to cancer (25). It has been 

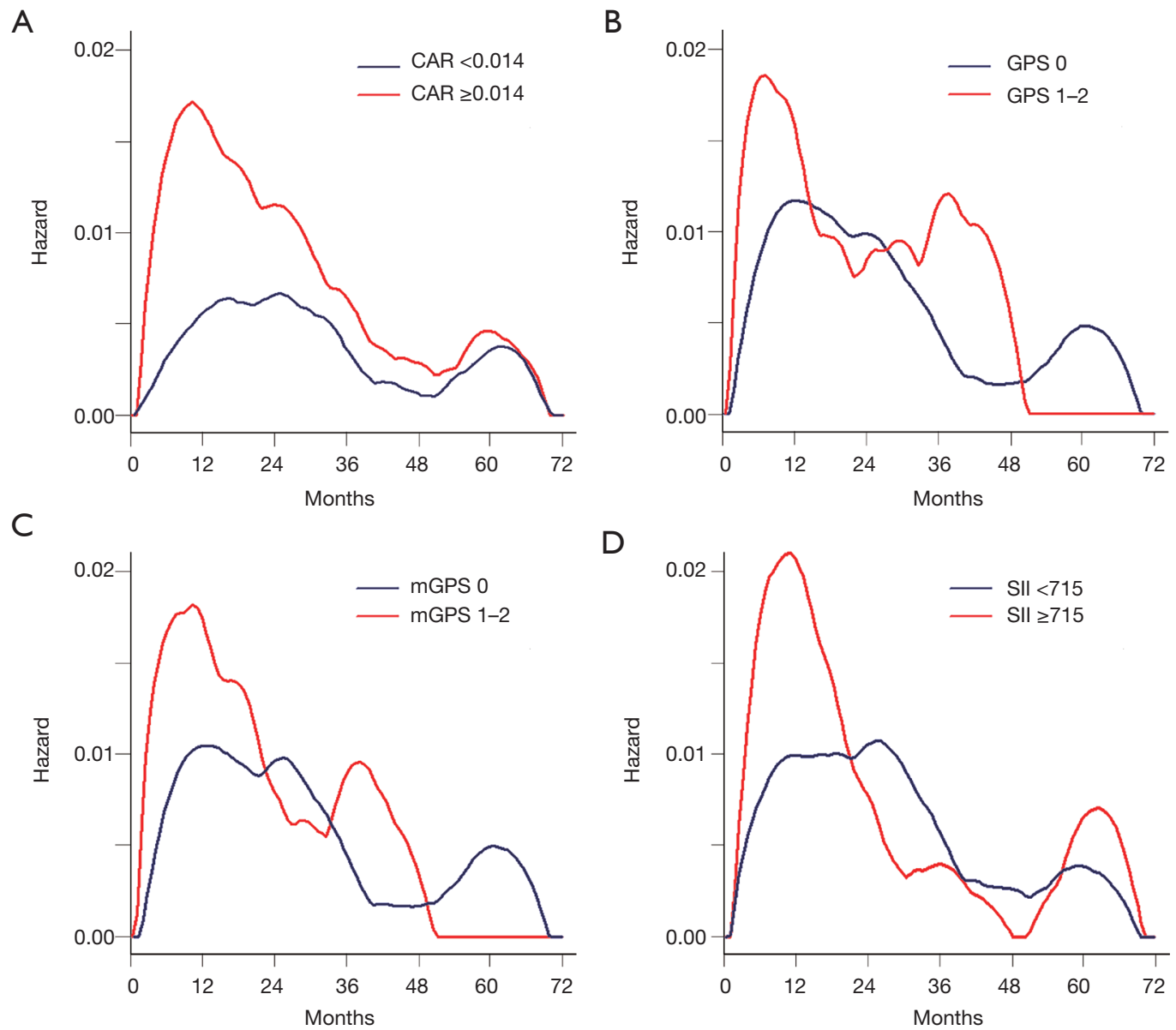

Figure 4 Smoothed hazard estimates according to inflammation-based scores. (A) CAR; (B) GPS; (C) mGPS; (D) SII. CAR, C-reactive protein-to-albumin ratio; GPS, Glasgow prognostic score; mGPS, modified GPS; SII, Systemic immune-inflammation index.

reported that the systemic inflammatory response may play a role in the development of cancer by inducing genetic instability, stimulating proliferative and survival signals, promoting invasion and metastatic spread, and enhancing local immunosuppression $(4,5,7,26)$.

Many studies have already shown that the presence of a systemic inflammatory response in cancer patients is associated with hypoalbuminemia and excessive weight loss, thus leading to a poor performance and reducing survival in various malignancies $(27,28)$. In this context, not only the CRP levels and neutrophil count but also serum albumin levels are strongly connected to systemic inflammation. Recent studies have revealed relationships between lung cancer and inflammation-based scores, including the CAR, SII, GPS, and mGPS, and have reported that such inflammation-based scores may be useful indicators of the prognosis in patients with resected NSCLC $(8,9,14-19)$.
Some studies have shown that an increased CRP level is related to primary tumor burden $(29,30)$, since the growth of cancer results in tissue damage, which disrupts the microenvironment and thereby induces inflammation (31). The poor RFS of patients with elevated CAR, SII, GPS, and mGPS in the present study might have been simply due to the high rate of patients with advanced-stage disease. To clarify this point, we therefore studied the hazard curves according to the pathological stage. As expected, the proportion of patients with elevated CAR, SII, and mGPS values was relatively low among stage I patients but increased among stage II or higher patients, and the hazard of recurrence peaked higher and earlier in patients with elevated CAR and SII values among cases of stage II or higher disease than those of stage I. Such an initial maximum peak during the first year indicated the aggressiveness of tumors with elevated CAR, SII, and GPS 
Table 2 Comparisons of inflammation-based scores and laboratory variables between the subgroups of pathological stage

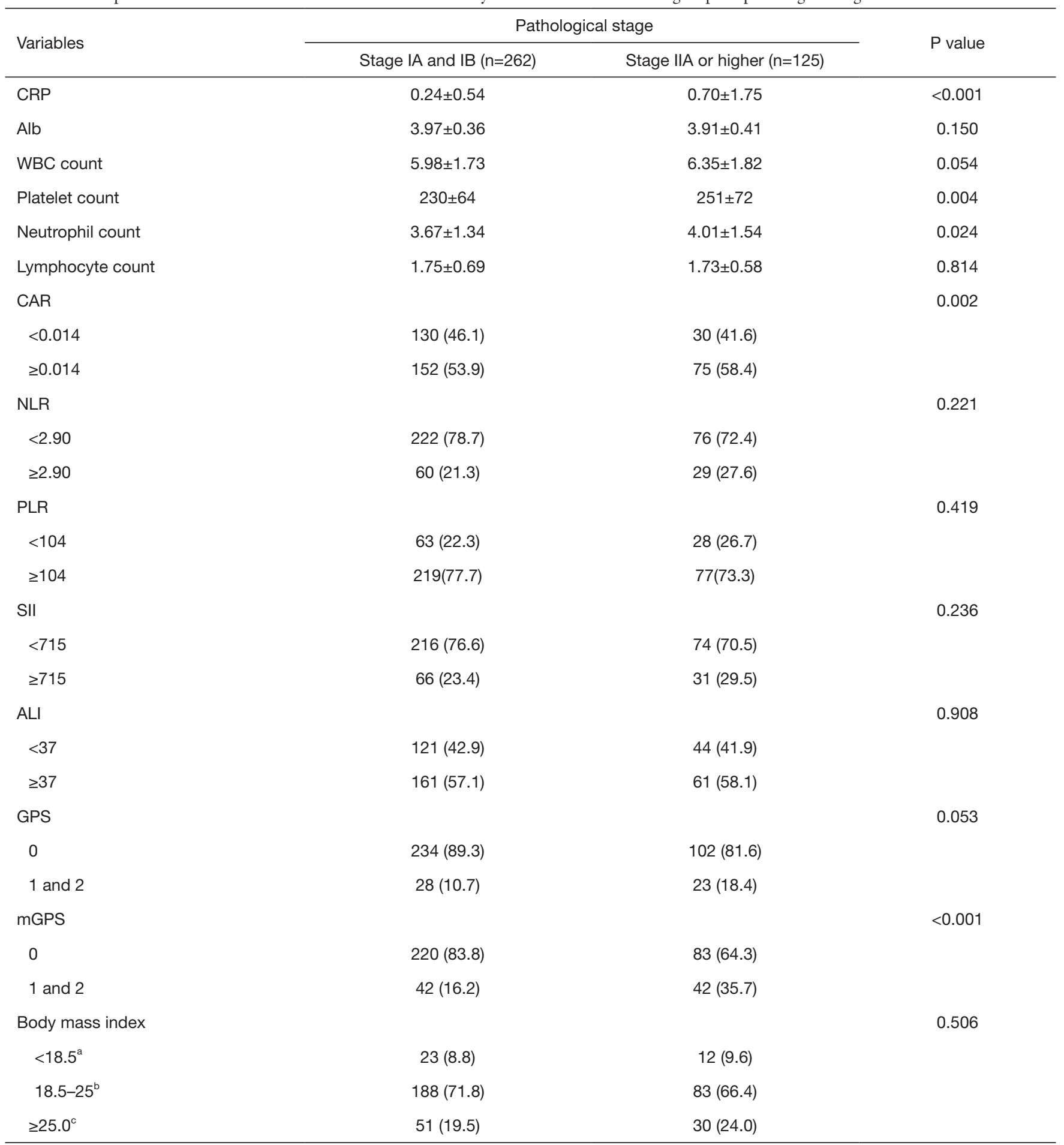

Data are presented as $\mathrm{n}(\%)$ or mean $\pm \mathrm{SD} .{ }^{\mathrm{a}}$, underweight. ${ }^{\mathrm{b}}$, normal weight. ${ }^{\mathrm{c}}$, overweight and obesity. Alb, albumin; ALI, advanced lung cancer inflammation index; BMI, body mass index; CAR, the C-reactive protein/albumin ratio; CRP, the C-reactive protein; GPS, Glasgow prognostic score; mGPS, modified GPS; NLR, neutrophil-lymphocyte ratio; PLR, platelet lymphocyte ratio; SII, Systemic immuneinflammation index; WBC, white blood cell; SD, standard deviation 
Table 3 Findings from multivariate analyses of RFS

\begin{tabular}{|c|c|c|c|}
\hline Variable & \multicolumn{3}{|c|}{ Multivariate } \\
\hline Age & 0.994 & $0.972-1.017$ & 0.609 \\
\hline Gender (male vs. female) & 1.834 & $0.987-3.410$ & 0.055 \\
\hline Body mass index & 0.951 & $0.890-1.016$ & 0.138 \\
\hline CAR $(\geq 0.014$ vs. $<0.014)$ & 1.987 & $1.202-3.284$ & 0.007 \\
\hline $\operatorname{PLR}(\geq 104$ vs. $<104)$ & 0.846 & $0.518-1.383$ & 0.505 \\
\hline SII ( $\geq 715$ vs. $<715)$ & 1.447 & $0.711-2.944$ & 0.308 \\
\hline GPS (0 vs. 1 and 2$)$ & 1.218 & $0.625-2.375$ & 0.562 \\
\hline $\operatorname{NLR}(\geq 2.90$ vs. $<2.90)$ & 1.251 & $0.596-2.628$ & 0.554 \\
\hline Surgical procedure (sublobar vs. lobectomy) & 0.884 & $0.509-1.535$ & 0.661 \\
\hline Surgery type (open vs. ATS) & 1.409 & $0.845-2.349$ & 0.189 \\
\hline Pathological stage (IIA or higher vs. I) & 4.430 & $0.987-3.410$ & $<0.001$ \\
\hline
\end{tabular}

ALI, advanced lung cancer inflammation index; CAR, the C-reactive protein/albumin ratio; GPS, Glasgow prognostic score; mGPS, modified GPS; PLR, platelet lymphocyte ratio; SII, Systemic immune-inflammation index; NLR, neutrophil-lymphocyte ratio; VATS, videoassisted thoracoscopic surgery.

values. Of particular note, patients with elevated CAR and SII values had an early maximum peak even after complete resection for stage I NSCLC, implying a high malignancy potential.

Although various inflammatory mediators such as inflammatory cytokines, vascular endothelial growth factor (VEGF), and prostaglandins reportedly play an important role in cancer progression (6-8), the mechanism underlying the relationship between systemic inflammation and early recurrence of cancer remains poorly understood. Furthermore, it is difficult to explain why there is a peak at all and why it comes so soon after complete resection. Possible explanations include that patients with elevated CAR, SII, GPS, and mGPS values have (I) tumors with an inherently more aggressive growth nature and (II) a decreased ability to inhibit micrometastases that have disseminated to different parts of the body during the preoperative period than those with low values. Regardless, the fact that the initial surge of recurrence develops so shortly after complete resection indicates that systemic inflammation triggered by surgical invasion disrupts the microenvironment, thus causing the production and secretion of various inflammatory mediators, thus accelerating the proliferation of tumor cells in previously undetectable metastatic lesions $(32,33)$.

Our results suggest the significance of focusing on the timing of recurrence as well as the total recurrence risk. It may therefore be effective to identify the sub-groups of patients who are most likely to develop early recurrence within a determined stage. At present, there is no consensus regarding the usefulness of postoperative follow-up (34), and tumor recurrence after complete resection remains the primary cause of cancer-related death. However, advances in molecular-targeted therapy and immunotherapy have improved the outcomes in patients with recurrent NSCLC. Although biologically aggressive, patients with recurrent NSCLC can be treated with drug therapy, if the presence of a driver mutation is confirmed. Therefore, it is reasonable to suppose that the early detection and treatment of recurrence may improve the patient survival rates and quality of life. In addition, based on our findings, it is reasonable to assume that patients with elevated CAR, SII, GPS, and mGPS values need more intensive surveillance than those with low values. Consequently, a method for analyzing the risk of postoperative recurrence based on a hazard curve is needed to accurately detect treatable recurrent diseases, thereby 
A

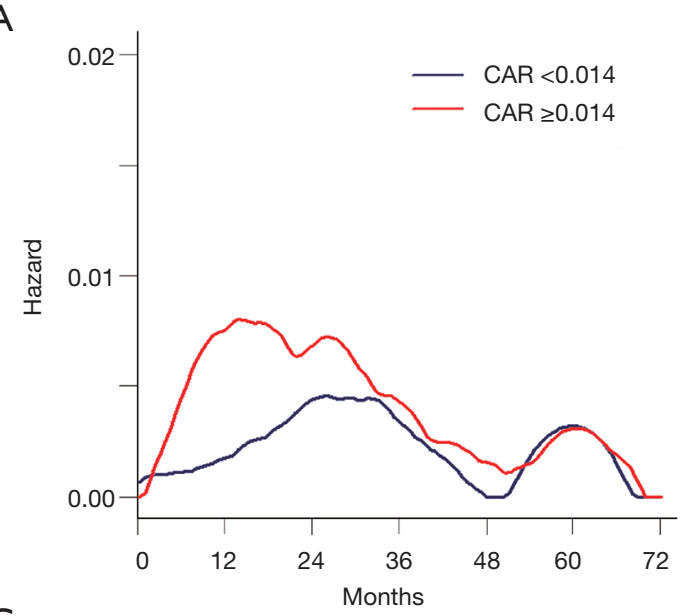

C

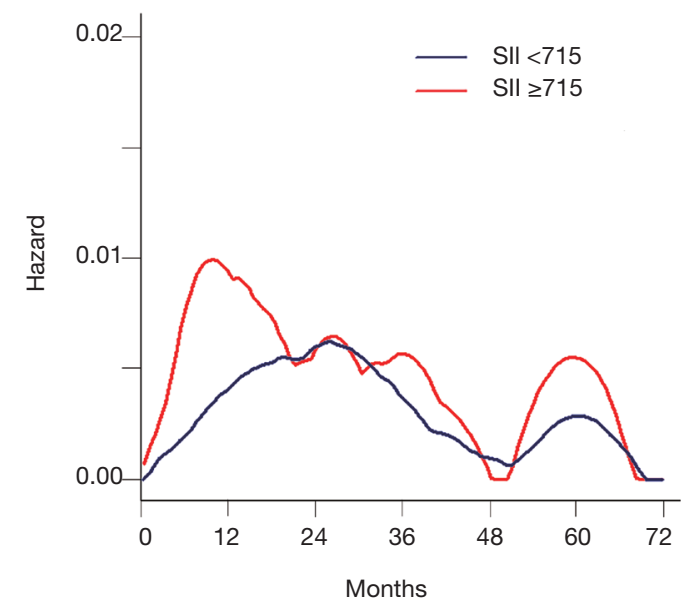

B

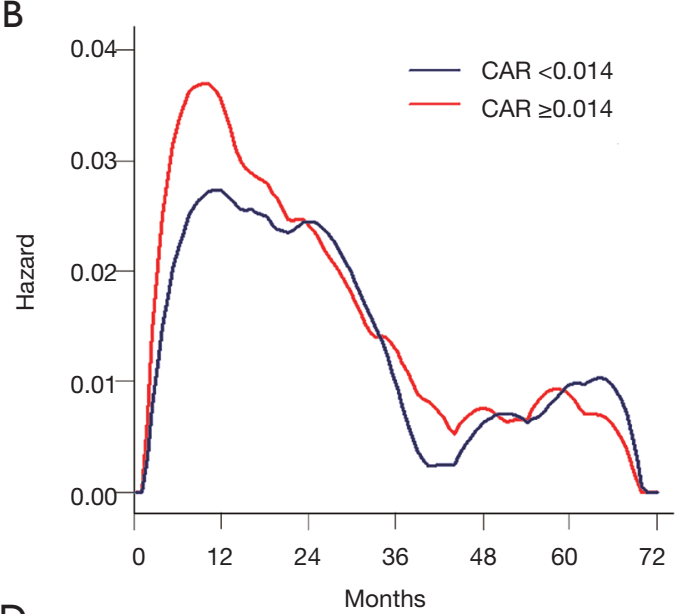

D

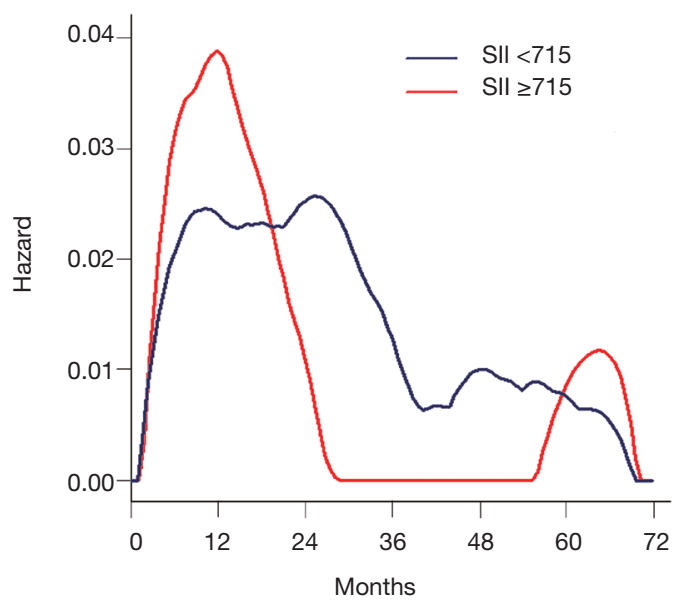

Figure 5 Smoothed hazard estimates of CAR (A,B) and SII (C,D) according to the pathological stage. (A) CAR, stage I; (B) CAR, stage IIA or higher; (C) SII, stage I; (D) SII, stage IIA or higher. CAR, C-reactive protein-to-albumin ratio; SII, Systemic immune-inflammation index.

minimizing the frequency of imaging studies.

The tumor histology, biomarker values [e.g., epidermal growth factor receptor (EGFR), anaplastic lymphoma kinase (ALK), and programmed death receptor 1 (PD-L1)], and postoperative TNM classification together represent the gold-standard for predicting the outcomes of NSCLC at present. However, these are tumor-related factors that are obtained after surgery. In contrast, inflammationbased scores are host-related factors that can be confirmed preoperatively and are independent of the postoperative findings. The CAR, GPS, and mGPS, calculated by means of the serum CRP and serum albumin levels, may reflect both the inflammatory condition and nutritional status. The SII is calculated using the following formula: platelets
× neutrophils / lymphocytes. An elevated SII usually suggests a higher level of inflammation and a lower level of immunity in patients (18). These inflammation-based scores are objective, simple, universally available, and costeffective measurements. Together with the postoperative histopathological findings, these preoperative inflammationbased scores represent a reliable supportive prognostic parameter for identifying patients at risk of early recurrence and tailoring the postoperative follow-up to individual patients with NSCLC.

The present study is associated with several limitations. First, this was a retrospective study with a limited number of patients and was performed at a single institution. Therefore, there may be a selection bias. Further 
prospective studies with larger populations therefore need to be carried out to determine the role of inflammationbased scores in predicting postoperative recurrence for NSCLC.

\section{Conclusions}

In conclusion, an elevated CAR value was significantly associated with a poor RFS, and patients with elevated CAR and SII values retain a high risk of early recurrence even after complete resection of stage I NSCLC. In addition, preoperative inflammation-based scores can be an objective, simple, and cost-effective measurements for predicting early recurrence.

\section{Acknowledgments}

Funding: None.

\section{Footnote}

Reporting Checklist: The authors have completed the REMARK reporting checklist. Available at http://dx.doi. org/10.21037/jtd-20-3458

Data Sharing Statement: Available at http://dx.doi. org/10.21037/jtd-20-3458

Peer Review File: Available at http://dx.doi.org/10.21037/jtd20-3458

Conflicts of Interest: All authors have completed the ICMJE uniform disclosure form (available at http://dx.doi. org/10.21037/jtd-20-3458). The authors have no conflicts of interest to declare.

Ethical Statement: The authors are accountable for all aspects of the work in ensuring that questions related to the accuracy or integrity of any part of the work are appropriately investigated and resolved. The study was conducted in accordance with the Declaration of Helsinki (as revised in 2013). The study was approved by the institutional ethics board of National Hospital Organization Yokohama Medical Center (No. 2019-11), and individual consent for this retrospective analysis was waived.

Open Access Statement: This is an Open Access article distributed in accordance with the Creative Commons
Attribution-NonCommercial-NoDerivs 4.0 International License (CC BY-NC-ND 4.0), which permits the noncommercial replication and distribution of the article with the strict proviso that no changes or edits are made and the original work is properly cited (including links to both the formal publication through the relevant DOI and the license). See: https://creativecommons.org/licenses/by-nc-nd/4.0/.

\section{References}

1. DeSantis CE, Lin CC, Mariotto AB, et al. Cancer treatment and survivorship statistics, 2014. CA Cancer J Clin 2014;64:252-71.

2. Demicheli R, Fornili M, Ambrogi F, et al. Recurrence dynamics for non-small-cell lung cancer: effect of surgery on the development of metastases. J Thorac Oncol 2012;7:723-30.

3. Watanabe K, Tsuboi M, Sakamaki K, et al. Postoperative follow-up strategy based on recurrence dynamics for non-small-cell lung cancer. Eur J Cardiothorac Surg 2016;49:1624-31.

4. Mantovani A, Allavena P, Sica A, et al. Cancer-related inflammation. Nature 2008;454:436-44.

5. Elinav E, Nowarski R, Thaiss CA, et al. Inflammationinduced cancer: crosstalk between tumours, immune cells and microorganisms. Nat Rev Cancer 2013;13:759-71.

6. Hanahan D, Weinberg RA. Hallmarks of cancer: the next generation. Cell 2011;144:646-74.

7. Diakos CI, Charles KA, McMillan DC, et al. Cancerrelated inflammation and treatment effectiveness. Lancet Oncol 2014;15:e493-503.

8. Yamauchi Y, Safi S, Muley T, et al. C-reactive proteinalbumin ratio is an independent prognostic predictor of tumor recurrence in stage IIIA-N2 lung adenocarcinoma patients. Lung Cancer 2017;114:62-7.

9. Zhang F, Ying L, Jin J, et al. The C-reactive protein/ albumin ratio predicts long-term outcomes of patients with operable non-small cell lung cancer. Oncotarget 2017;8:8835-42.

10. Sarraf KM, Belcher E, Raevsky E, et al. Neutrophil/ lymphocyte ratio and its association with survival after complete resection in non-small cell lung cancer. J Thorac Cardiovasc Surg 2009;137:425-8.

11. Gu XB, Tian T, Tian XJ, et al. Prognostic significance of neutrophil-to-lymphocyte ratio in non-small cell lung cancer: a meta-analysis. Sci Rep 2015;5:12493.

12. Zhang H, Gao L, Zhang B, et al. Prognostic value of platelet to lymphocyte ratio in non-small cell lung 
cancer: a systematic review and meta-analysis. Sci Rep 2016;6:22618.

13. Toda M, Tsukioka T, Izumi N, et al. Platelet-tolymphocyte ratio predicts the prognosis of patients with non-small cell lung cancer treated with surgery and postoperative adjuvant chemotherapy. Thorac Cancer 2018;9:112-9.

14. Forrest LM, McMillan DC, McArdle CS, et al. A prospective longitudinal study of performance status, an inflammation-based score (GPS) and survival in patients with inoperable non-small-cell lung cancer. Br J Cancer 2005;92:1834-36.

15. Jin J, Hu K, Zhou Y, et al. Clinical utility of the modified Glasgow prognostic score in lung cancer: A meta-analysis. PLoS One 2017;12:e0184412.

16. Yotsukura M, Ohtsuka T, Kaseda K, et al. Value of the Glasgow prognostic score as a prognostic factor in resectable non-small cell lung cancer. J Thorac Oncol 2016;11:1311-8.

17. Osugi J, Muto S, Matsumura Y, et al. Prognostic impact of the high-sensitivity modified Glasgow prognostic score in patients with resectable non-small cell lung cancer. J Cancer Res Ther 2016;12:945-51.

18. Tomita M, Ayabe T, Maeda R, et al. Systemic immuneinflammation index predicts survival of patients after curative resection for non-small cell lung cancer. In Vivo 2018;32:663-7.

19. Guo W, Cai S, Zhang F, et al. Systemic immuneinflammation index (SII) is useful to predict survival outcomes in patients with surgically resected non-small cell lung cancer. Thoracic Cancer 2019;10:761-8.

20. Jafri SH, Shi R, Mills G. Advance lung cancer inflammation index (ALI) at diagnosis is a prognostic marker in patients with metastatic non-small cell lung cancer (NSCLC): a retrospective review. BMC Cancer 2013;13:158.

21. Goldstraw P, Crowley J, Chansky K, et al. The IASLC Lung Cancer Staging Project: proposals for the revision of the TNM stage groupings in the forthcoming (seventh) edition of the TNM Classification of malignant tumours. J Thorac Oncol 2007;2:706-14. Erratum in: J Thorac Oncol
2007;2:985.

22. Martini N, Melamed MR. Multiple primary lung cancers. J Thorac Cardiovasc Surg 1975;70:606-12.

23. Shen KR, Meyers BF, Larner JM, et al. Special treatment issues in lung cancer: ACCP evidencebased clinical practice guidelines ( 2 nd edition). Chest 2007;132:290S-305S.

24. Müller HG, Wang JL. Hazard rate estimation under random censoring with varying kernels and bandwidths. Biometrics 1994;50:61-76.

25. Balkwill F, Mantovani A. Inflammation and cancer: Back to Virchow? Lancet 2001;357:539-45.

26. Colotta F, Allavena P, Sica A, et al. Cancer-related inflammation, the seventh hallmark of cancer: links to genetic instability. Carcinogenesis 2009;30:1073-81.

27. McMillan DC. The systemic inflammation-based Glasgow prognostic score: a decade of experience in patients with cancer. Cancer Treat Rev 2013;39:534-40.

28. Roxburgh CSD, McMillan DC. Cancer and systemic inflammation: treat the tumour and treat the host. Br J Cancer 2014;110:1409-12.

29. Alifano M, Falcoz PE, Seegers V, et al. Preresection serum C-reactive protein measurement and survival among patients with resectable non-small cell lung cancer. J Thorac Cardiovasc Surg 2011;142:1161-67.

30. Lee JG, Cho BC, Bae MK, et al. Preoperative C-reactive protein levels are associated with tumor size and lymphovascular invasion in resected non-small cell lung cancer. Lung Cancer 2009;63:106-10.

31. Hwang EC, Hwang IS, Yu HS, et al. Utility of inflammation-based prognostic scoring in patients given systemic chemotherapy first-line for advanced inoperable bladder cancer. Jpn J Clin Oncol 2012;42:955-60.

32. Hedley BD, Chambers AF. Tumor dormancy and metastasis. Adv Cancer Res 2009;102:67-101.

33. Tohme S, Simmons RL, Tsung A. Surgery for cancer: a trigger for metastases. Cancer Res 2017;77:1548-52.

34. Schmidt-Hansen M, Baldwin DR, Hasler E. What is the most effective follow-up model for lung cancer patients? A systematic review. J Thorac Oncol 2012;7:821-4.
Cite this article as: Watanabe K, Noma D, Masuda H, Masuda M. Preoperative inflammation-based scores predict early recurrence after lung cancer resection. J Thorac Dis 2021;13(5):2812-2823. doi: 10.21037/jtd-20-3458 